\title{
The role of the surgeon in the management of oligometastatic non-small cell lung cancer: a literature review
}

\author{
Lawek Berzenji, Sophie Debaenst, Jeroen M. H. Hendriks, Suresh Krishan Yogeswaran, Patrick Lauwers, \\ Paul E. Van Schil \\ Department of Thoracic and Vascular Surgery, Antwerp University Hospital, Edegem, Belgium \\ Contributions: (I) Conception and design: L Berzenji, S Debaenst, PE Van Schil; (II) Administrative support: SK Yogeswaran, P Lauwers, JMH \\ Hendriks; (III) Provision of study materials or patients: None; (IV) Collection and assembly of data: L Berzenji, S Debaenst, PE Van Schil; (V) Data \\ analysis and interpretation: All authors; (VI) Manuscript writing: All authors; (VII) Final approval of manuscript: All authors. \\ Correspondence to: Paul E. Van Schil, MD, PhD. Department of Thoracic and Vascular Surgery, Antwerp University Hospital, Drie Eikenstraat 655, \\ B-2650 Edegem (Antwerp), Belgium. Email: paul.van.schil@uza.be.
}

Objective: In this review, we aim to summarize the most recent data on the surgical management of oligometastatic non-small cell lung cancer (NSCLC).

Background: Approximately $60-70 \%$ of all patients with NSCLC initially present with advanced stages of cancer at time of diagnosis. These patients are generally treated with chemotherapy, radiation therapy, or a combination of these modalities. Patients with late-stage disease are usually not considered to be amenable for curative-intent treatments due to poor prognoses. Despite advances in systemic therapies, 5-year overall survival rates in these patients remain poor. However, technological advances in imaging modalities and new imaging strategies have substantially increased tumor detection rates and have resulted in a shift towards earlier diagnosis of NSCLC, possibly in stages in which metastatic disease is limited and still treatable. Studies in recent years have shown that there is a distinct group of patients with metastatic lesions at one or a few sites, often referred to as oligometastatic disease, that may have better survival outcomes compared to patients with more disseminated diseases. Furthermore, it is suggested that these patients may benefit from a combination of systemic treatment and local treatment aimed at the metastatic site(s). However, the role of surgery in this setting remains a controversial subject, with many unanswered questions.

Methods: The PubMed/MEDLINE database and the Cochrane database were searched to find relevant articles regarding oligometastatic NSCLC. Specifically, articles regarding definitions of oligometastatic disease, oligometastatic tumor biology, diagnosis, and the treatment of oligometastatic disease were identified.

Conclusions: Oligometastatic NSCLC represents a wide spectrum of diseases and encompasses a heterogeneous patient population. Current data suggests that local ablative treatment of oligometastatic lesions with surgery or stereotactic body radiation therapy may result in improved overall survival and progression-free survival rates. However, more data from multi-center prospective trials are necessary to shed light on which therapeutic modalities are most suitable for the treatment of oligometastatic NSCLC. Integration of clinical and molecular staging data is necessary to allow for more personalized treatment approaches.

Keywords: Oligometastasis; non-small cell lung cancer (NSCLC); surgery; local therapy

Submitted Jan 25, 2021. Accepted for publication Jul 23, 2021.

doi: $10.21037 /$ tlcr-21-58

View this article at: https://dx.doi.org/10.21037/tlcr-21-58 


\section{Introduction}

Lung cancer is worldwide the second most common type of cancer and one of the leading causes of cancer-related deaths (1). In Europe, non-small cell lung cancer (NSCLC) accounts for approximately $85 \%$ of all lung cancer diagnoses, and around $60-70 \%$ of these patients present with advanced stages at time of diagnosis $(2,3)$. Generally, patients with late-stage NSCLC are treated with systemic or palliative therapies, including chemotherapy, radiation therapy (RT), or both. The 5-year overall survival (OS) rates of NSCLC are $26 \%$ for stage IIIb, $10 \%$ for stage IVa, and $1 \%$ for stage IVb (4). Previously, patients with these advances stages of diseases were not considered to be amenable for curative-intent treatments due to their poor prognoses. However, advances in imaging modalities and screening strategies in more recent years have substantially increased tumor detection rates and have resulted in a shift towards earlier diagnosis of NSCLC (5). Furthermore, the identification of new molecular alterations and the discovery of their respective targeted treatments, such as for anti-epidermal growth factor receptor (EGFR), anaplastic lymphoma kinase (ALK), and ROS1, have resulted in an almost exponential emergence of new treatment modalities for advanced stages of NSCLC (6). When applied in carefully selected treatment populations, these treatments can provide major improvements in disease-free survival (DFS) and OS rates in these patients (6-8).

These developments have led to an increasing number of patients with a limited number of metastatic lesions at only a few sites, often referred to as oligometastatic disease (9). This is also reflected in the $8^{\text {th }}$ edition of the Tumor, Node, Metastasis (TNM) classification published by the International Association for the Study of Lung Cancer (IASLC) which included oligometastatic disease as a separate category for the first time since its introduction. In this latest TNM classification, patients with metastatic disease are divided into three distinct subgroups: stage M1a: involvement of the lung alone; M1b: single extrathoracic metastasis; and M1c: multiple extrathoracic metastases in one or more organs (10). Recent studies have suggested that patients with oligometastatic disease have better OS rates compared to patients with more disseminated diseases, and that they may benefit from a combination of systemic treatment and local treatment aimed at the metastatic site(s) (5,11-13). However, the specific role of surgical therapies in the treatment of oligometastatic disease remains a controversial and challenging subject with many unanswered questions regarding topics such as longterm treatment outcomes and patient selection criteria. In this review, we aim to summarize the most recent and relevant data on the surgical management of oligometastatic NSCLC. We present the following article in accordance with the Narrative Review reporting checklist (available at https://dx.doi.org/10.21037/tlcr-21-58).

\section{Methods}

The PubMed/MEDLINE database and the Cochrane database were searched to identify relevant articles regarding oligometastatic NSCLC. Specifically, articles regarding definitions of oligometastatic disease, oligometastatic tumor biology, diagnosis, and the treatment of oligometastatic disease were identified. Narrative reviews, clinical trials, systematic reviews, meta-analyses, and guidelines written in English and from the period of 2015-2021 were included in this study. Studies published before 2015 that were deemed vital to the review were also included.

\section{Definition of oligometastatic disease}

The term "oligometastasis" is a composite derived from the Greek words "oligo", meaning "a few" and "metastasis", meaning "removal" or migration". In 1995, Hellman and Weichselbaum introduced the term oligometastasis to refer to a state of limited systemic metastatic disease in which local treatments could be curative (14). This resulted in a paradigm shift where cancer metastasis was no longer viewed as a binary concept in which a tumor is either localized and curable, or disseminated and, by definition, incurable. Rather, the behavior of metastatic disease is more likely to represent a spectrum in which, initially, patients have limited disease in one or a few sites before the metastases becomes more disseminated (15). This spectrum theory of tumor metastasis posits that differences in metastatic potential are rooted in the molecular features of cancers which determine its metastatic virulence. Many of these features were not characterized yet at the time of Hellman and Weichselbaum's first description of oligometastatic disease. However, more recent studies have identified several biological mechanisms that support this theory (16).

A number of molecular markers have been proposed to play a significant role in the differentiation between oligometastatic and polymetastatic disease. For example, analysis of microRNA expression in cancer patients has 
allowed the identification of potential "oligomiRs" that could help identify oligometastatic phenotypes (17). In a study performed by Lussier et al., the authors analyzed patterns of microRNA expression in tumor samples from patients with oligometastatic disease that were treated with high-dose RT. Their study results showed that patients who did not develop polymetastases were characterized by unique features of microRNA-200c, a top prioritized microRNA (18). Earlier studies have shown that several members of the microRNA-200 family are involved in metastatic disease (19). Lussier et al. demonstrated that microRNA-200c enhancement in an oligometastatic cell line resulted in conversion from oligo- to polymetastases. In a subsequent study by Lussier et al., the authors investigated the role of microRNA expression patterns in patients with oligometastatic NSCLC patients ( $<5$ initial metastases) that were treated with curative-intent surgery. Their results showed that microRNA expression patterns were distinctly different between patients with high and low rates of progression. Furthermore, these prioritized microRNAs were associated with rate of progressive disease and survival in an independent dataset. The authors concluded that oligo- and polymetastatic disease are distinct entities, both at clinical and a molecular level (20). Additional studies have shown that predicted target genes for a number of oligomiRs are involved in the molecular pathways that are vital to tumor adhesion, epithelial-mesenchymal transition, invasion, and migration $(21,22)$. Other molecular features that may determine metastatic potential include copy number alterations (CNA), driver mutations, and intratumor heterogeneity (16).

Studies have suggested that cancer biology can be variable between patients, with some cancers progressing more slowly than others, resulting in variable progression patterns over time $(23,24)$. These variations in progression phenotypes may be associated with different expression patterns in microRNA (20). The incidence of oligometastases in patients with NSCLC is estimated to range between $26-50 \%$, depending on which definition is used (i.e., number of metastatic lesions and oligometastatic subtype) (25). In NSCLC, the most common (oligo) metastatic site is the brain, accounting for approximately $35.5 \%$, followed by multi-organ metastases to the contralateral lung $(33.6 \%)$, adrenal glands $(10 \%)$, bone $(8.5 \%)$, and the liver (2.4\%) (11). Several subtypes of oligometastatic disease have been described, which are classified according to the initial diagnosis and/or their response to systemic therapy. In synchronous or "de novo" oligometastasis, patients present with oligometastases at the time of initial detection of the primary tumor. In contrast, metachronous oligometastatic disease refers to the development of a limited number of metastases after initial diagnosis and treatment of the primary tumor (26). Two other terms that are frequently used in this setting are "oligoprogression" and "oligopersistence". While the first two terms are entities that are more closely related to tumor biology, oligoprogression and oligopersistence are generally used to describe oligometastases that are induced as a result of treatment with systemic therapies such as targeted therapies, immunotherapy, and, sometimes, chemotherapy (Figure 1). Patients treated with tyrosine kinase inhibitors (TKIs) are particularly susceptible to oligoprogression and oligopersistence due to acquired resistance through phenotypic transformation. In oligoprogression, patients develop a limited number of new metastases in one or a few sites after an initial favorable response to systemic treatments, usually targeted therapy, immunotherapy, or a combination of chemo- and immunotherapy. Often, progression in these cases occurs at a limited number of anatomic sites. It has been proposed that these patterns of progression are caused by intratumoral and intertumoral genetic heterogeneity. Oligopersistence refers to a situation in which patients have residual metastatic disease at a few sites after a favorable response to initial systemic therapy (15). Table 1 shows an overview of the most important definitions regarding oligometastatic disease.

Currently, there is no consensus on which threshold should be used to define oligometastatic disease in terms of the number of sites and lesions involved. There is a wide range of different eligibility criteria used in studies involving oligometastatic disease, both in terms of sites/ organs involved and the number of lesions. However, in the majority of the available trials, a large proportion of patients enrolled have only 1 or 2 lesions, even in studies where more than 1-2 lesions are permitted. This suggests that investigators in these trials favor patients with fewer lesions. Furthermore, this means that the limited outcome data that is available is mainly based on treatment outcomes of patients with just 1 or 2 lesions $(27,28)$. In a systematic review by Giaj-Levra et al., the authors found no consensus with regards to a definition of oligometastatic disease, however, the majority of studies included in their analysis adopted a threshold of no more than five lesions (29). This heterogeneity makes it quite difficult to create evidence-based guidelines for oligometastatic disease. To overcome these difficulties, the European Organization of 
A

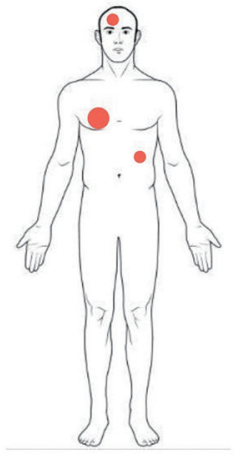

C

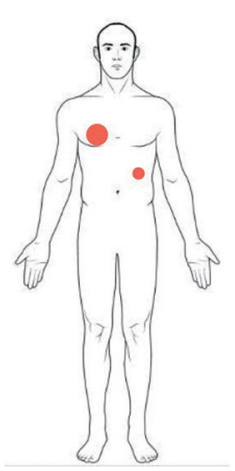

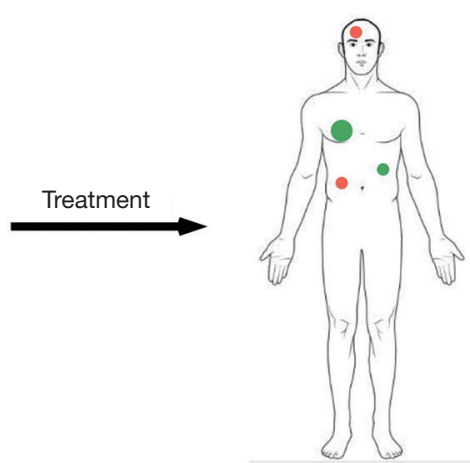

B
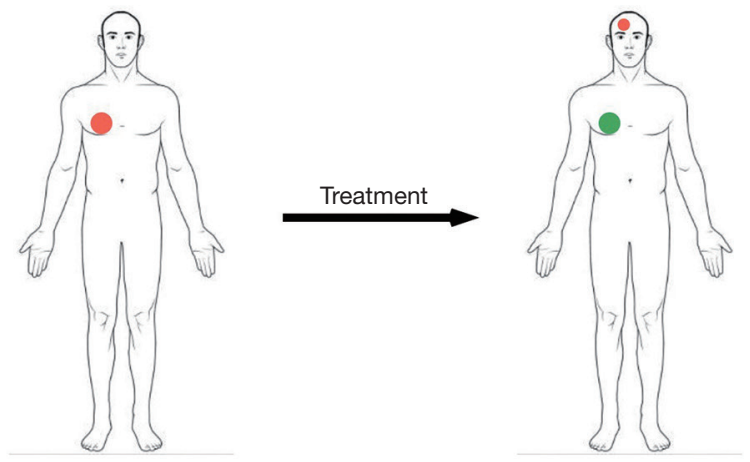

D
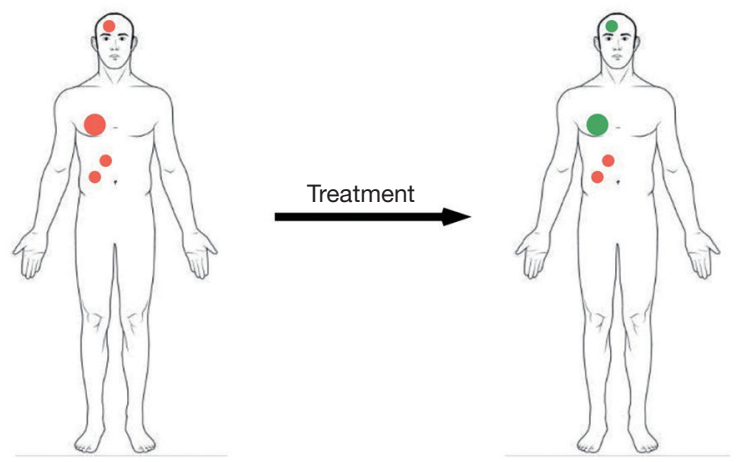

Figure 1 Overview of different subtypes of oligometastatic disease in NSCLC. (A) Synchronous oligometastatic disease with the primary lung tumor and metastatic lesions appearing at the same time; (B) metachronous oligometastatic disease with a metastatic (brain) lesion appearing after initial treatment for the primary lung tumor; (C) oligoprogression with new metastatic lesions appearing after initial treatment for oligometastatic NSCLC; (D) oligopersistence with persistent metastatic lesions after initial treatment for oligometastatic NSCLC. NSCLC, non-small cell lung cancer.

Table 1 Definitions of different terms related to oligometastatic diseases

\begin{tabular}{ll}
\hline Term & Definition \\
\hline Oligometastasis & A state of limited systemic metastatic disease in which local therapies could be curative \\
Synchronous oligometastatic disease & Metastasis present at the time of diagnosis of primary tumor \\
Metachronous oligometastatic disease & Metastasis detected separately after an interval of time (not further specified) \\
Oligopersistence & Persistent oligometastatic disease after initial treatment \\
Oligoprogression & Progressive oligometastatic disease after initial treatment \\
\hline
\end{tabular}

Research and Treatment of Cancer (EORTC) established a pan-European multidisciplinary group with the goal of developing a consensus definition of oligometastatic disease. In their published consensus findings, the authors explained that the maximum number of lesions and organs involved depend on whether it is possible to offer a radical intent treatment strategy to the patient. They further stated that, based on their data, a maximum of five metastatic lesions and three organs are used as definition for oligometastatic NSCLC. In addition, the authors clarified that all organs are allowed, except for bone marrow involvement and diffuse serosal metastases (meningeal, pericardial, pleural, and mesenteric), due to the fact that these metastases cannot be treated with radical intent (30).

In order to accurately identify oligometastatic disease, a careful and complete radiological work-up is necessary. In addition to standard computed tomography (CT) scans, a 
large number of studies advocate the use of brain magnetic resonance imaging (MRI) and ${ }^{18} \mathrm{~F}$-fluorodeoxyglucose positron emission tomography $\left({ }^{18} \mathrm{~F}-\mathrm{FDG} \mathrm{PET}\right)$ in the staging of NSCLC. This is also the general consensus in the publications of major scientific societies such as the European Society for Medical Oncology (ESMO), National Comprehensive Cancer Network (NCCN), and EORTC $(9,29,31,32)$. Earlier data has shown that MRI is superior to CT for detecting brain metastases (33). Furthermore, ${ }^{18} \mathrm{~F}$-FDG PET/CT-scans are generally used to diagnose systemic disease and mediastinal lymph node status, with sensitivity and specificity rates ranging between $79-85 \%$ and $87-92 \%$, respectively (34). The additional use of ${ }^{18} \mathrm{~F}$-FDG PET/CT-scans is associated with improved treatment outcomes as stage migration may occur. This is due to higher rates of metastasis detection with PET/CT compared to the use of CT-scans alone. It is estimated that around $15 \%$ of all NSCLC patients initially staged as stage I-III with conventional CTimaging, are upstaged to stage IV with PET/CT $(9,35)$. This upstaging can obviate the use of aggressive local therapies in patients that are not likely to benefit from them. In a retrospective study by Tönnies et al., patients who underwent preoperative staging using ${ }^{18}$ F-FDG PETCT scans were compared to patients that were staged using conventional spiral CT-scans. The authors found a significant difference in 5-year survival rates, with an OS of $58 \%$ in the PET/CT group, compared to $33 \%$ in the CT group (36).

There is still some controversy regarding mediastinal staging and the need for pathological confirmation. Most studies agree on the fact that PET-CT is required for mediastinal lymph node staging, however, the issue of pathological confirmation is still hotly debated $(29,30)$. The role of mediastinal node involvement in oligometastatic disease is still not clear and it is still uncertain whether a positive mediastinal lymph node status should be viewed as metastatic disease. In the $8^{\text {th }}$ TNM classification, involvement of mediastinal lymph nodes is defined as locally advanced disease, and not as metastasis (4). The EORTC consensus group clarifies that pathological confirmation of mediastinal nodes is only indicated if it influences treatment strategy. In addition, the EORTC states that pathological confirmation of at least one metastatic lesion is required, especially in patients with a solitary metastasis or if the results may change the treatment strategy. However, it is required that the benefits of the pathological confirmation outweigh the risks (30).

\section{Surgical treatment of oligometastatic disease}

According to the EORTC consensus group, the main goal in the treatment of oligometastatic disease is to gain long-term disease control using a radical treatment that is technically feasible and has acceptable toxicity. The term "cure" was not used as the authors stated that patients could still benefit from radical therapies resulting in long-term disease control, even if they are not cured. However, technical feasibility is an important element in this definition, due to the fact that radical treatment may not be possible due to the location of the metastatic lesion or the patient's comorbidities, even in cases with a limited number of metastatic lesions. The type of radical treatment is not included in this definition, only the feasibility. As a result, genomic background and histologic subtype are not taken into account as well (30). One of the most important elements in the treatment selection for oligometastatic NSCLC is to identify patients that are most likely to benefit from radical metastasis-directed treatments. Only a relatively small subset of oligometastatic patients (15-25\%) will have an extended disease-free interval (DFI) after local treatment of metastases, thus necessitating careful patient selection in order to prevent administering futile therapies. Several prognostic factors associated with survival have been identified, such as: age, sex, number of metastases; involvement of mediastinal lymph nodes, DFI, tumor histology, performance status, pathological $\mathrm{T}$ stage, location of the metastasis/metastases, treatment type of the primary lesion, and the biomolecular profile $(9,37)$.

Traditionally, surgical treatment has been the main modality in oligometastatic NSCLC patients, with approximately $55 \%$ of all patients receiving surgical treatment (38). The indications for surgical treatment depend on a number of metastasis-related factors such as the number, size, and location of metastases, and on patient-specific factors such as age, performance status, comorbidities, and prognosis $(9,25,26)$. Both brain and adrenal oligometastases have shown to have relatively good prognoses after radical treatment with surgery, with 5 -year OS rates of approximately $20 \%$ and $20-30 \%$, respectively (39). The 2013 American College of Chest Physicians (ACCP) guidelines state that in patients with a synchronous resectable cN0,1 primary NSCLC and an isolated brain or adrenal metastasis with no other metastatic sites, resection of the primary lesion and the isolated brain/ adrenal metastasis is advised (40). Furthermore, the NCCN guidelines recommend local therapy for patients with 
oligometastatic disease, and multidisciplinary treatments (including systemic therapies) for primary lesions in patients with isolated brain metastases (41). However, clinicians should be aware that these guidelines are mostly based on expert opinions than on high quality data, and can thus include bias.

There are two main surgical treatment approaches for treating oligometastatic NSCLC. The first approach involves an initial resection of the primary tumor, followed by control of distant lesions using surgery or RT, and control of micrometastases using systemic therapy. The second approach involves initial treatment with systemic therapy, followed by adjuvant local treatment with surgery or RT for those patients that have responded to drug therapy, but have residual, localized tumors. The latter approach is often called the salvage (surgery) approach (42). In a study by Wang et al., the effect of initial surgical resection of the primary lesion in oligometastatic NSCLC was analyzed. In their retrospective study, 172 NSCLC patients with oligometastatic disease were divided into two groups: one group received primary surgical treatment followed by adjuvant chemotherapy, while the other group received neoadjuvant chemotherapy followed by local RT. Median survival times in the primary surgery group and neoadjuvant therapy group were 48 months and 18 months, respectively. Furthermore, 5-year OS rates were $21.1 \%$ and $7.6 \%$, respectively. The authors concluded that upfront surgical treatment of primary tumors significantly increased median survival times and 5-year OS in patients with oligometastatic NSCLC (43). However, it should be noted that selection bias is expected in this retrospective study. For example, patients in the neoadjuvant chemotherapy group may have had bulkier diseases than the primary surgery group.

Treatment outcomes of a salvage surgery approach in oligometastatic NSCLC were evaluated in a relatively recent phase II, multi-center, randomized controlled trial (RCT) by Gomez et al. Patients with pathological confirmation of stage IV NSCLC and three or fewer metastases after first-line systemic therapy were included. The sites of oligometastatic lesions in this study were: brain [10], bones [10], adrenal gland [8], pleura [7], lung [6], cervical lymph node [4], liver [2], spleen [2], retroperitoneal lymph node [1], paraspinal [1], and kidney [1]. The firstline therapy consisted of $\geq 4$ cycles of platinum doublet therapy or $\geq 3$ months of either EGFR or ALK inhibitors for patients that the respective mutations. After inclusion, patients were randomized and assigned to either local consolidative treatment (RT or surgical resection of all metastatic lesions) with or without subsequent maintenance treatment, or to maintenance treatment alone. The type of maintenance treatment was chosen by the treating physician(s) and included pemetrexed, bevacizumab, erlotinib, crizotinib, and observation (close surveillance without any cytotoxic therapies). The trial was terminated early after randomization of 49 patients ( 25 patients in the local consolidative therapy arm and 24 in the maintenance treatment arm) after an interim analysis demonstrated that the local consolidative arm extended progression-free survival (PFS) when compared to the maintenance therapy alone. The median PFS in the local consolidative therapy arm was 11.9 months, versus 3.9 months in the maintenance treatment arm. Of the 25 patients in the local consolidative therapy arm, six patients (24\%) received a combination of surgery and RT, and one patient (4\%) received surgery to all sites. No grade 3 or 4 toxicities were reported in this study. The authors concluded that, in patients with three or fewer NSCLC metastases, aggressive local therapy with or without maintenance therapy resulted in improved PFS rates compared to maintenance treatment alone (12).

Despite an increasing amount of research in the field of NSCLC, there is a lack of data on the outcomes of local consolidative therapy in the setting of oligoprogressive disease. However, the limited amount of evidence that is available supports the notion that local treatment of one or a few sites of progression in patients that have demonstrated a good response to systemic treatment is feasible, safe, and associated with favorable treatment outcomes (15). In a retrospective analysis by $\mathrm{Yu}$ et al., a total of 18 patients with oligoprogression after EGFR TKI therapy received local consolidative therapy (surgery, radiofrequency ablation, or stereotactic RT). Of the 18 patients, 11 (61\%) underwent surgery (wedge resection in 7 patients, lobectomy in 3 patients, and a pneumonectomy in 1 patient). Local therapy was well tolerated in all patients, and $85 \%$ of patients were able to restart TKI therapy within 1 month after the local therapy. Furthermore, median time to progression after local therapy was 10 months, and the median time until a subsequent change in systemic treatment was 22 months. Median OS from local therapy was 41 months. The authors concluded that local consolidative therapy followed by continuation of EGFR TKI therapy is well tolerated and associated with long OS and PFS rates (44). A study by Weickhardt et al. regarding EGFR- or ALKpositive NSCLC showed similar results, with a median time to progression after local therapy of 6.2 months (45). 
Table 2 Overview of ongoing trials using SBRT and/or surgery as treatment for oligometastatic disease

\begin{tabular}{|c|c|c|c|c|c|}
\hline Clinical trial & Phase & Intervention & Control & Estimated enrollment & Primary endpoint \\
\hline NCT02975609 & II & $\mathrm{CT}+\mathrm{SBRT}$ & $\mathrm{CT}+$ conventional RT & 100 & PFS \\
\hline NCT04908956 & II & Osimertinib + SBRT & Osimertinib & 60 & Safety + efficacy \\
\hline NCT03965468 & II & Durvalumab + CT + RT + surgery & - & 47 & PFS \\
\hline NCT02417662 & III & $\mathrm{SACT}+$ conventional RT (primary tumor) + SBRT & SACT & 340 & OS \\
\hline NCT04306926 & II & TQB2450 (anti-PD-L1) + SBRT & - & 59 & PFS \\
\hline NCT04758481 & $\mathrm{I} / \mathrm{II}$ & $\mathrm{RT}$ (primary tumor) + SBRT + maintenance RT & - & 20 & Toxicity + PFS \\
\hline NCT04486287 & II & Sintilimab + SBRT & - & 44 & ORR \\
\hline NCT03705403 & II & SBRT/RT + immunocytokine L19-IL2 & SOC & 126 & PFS \\
\hline NCT04767009 & II & SBRT + anti-PD-1 & - & 59 & AEs + LFS \\
\hline
\end{tabular}

SBRT, stereotactic body radiation therapy; PFS, progression-free survival; CT, chemotherapy; RT, radiation therapy; SACT, systemic anticancer therapy; OS, overall survival; ORR, objective response rate; SOC, standard of care; AEs, adverse events; LFS, lesion-free survival.

\section{Future perspectives}

In recent years, there has been a significant increase in the use of less invasive ablative techniques such as stereotactic body radiation therapy (SBRT) (25). Technological advances in imaging and radiation technologies have made it feasible to administer high ablative doses of RT with precision, without damaging surrounding tissues. These high-dose treatments can be given in fewer fractions, resulting in shorter treatment durations than conventional RT schedules. SBRT is currently mainly used for (oligo)metastatic lesions that are unresectable or for patients that are deemed unfit for surgical treatment due to comorbidities $(46,47)$. However, the notion that SBRT is only suitable for unresectable oligometastatic lesions or patients that are medically unfit for surgical treatment is erroneous. Several studies have been published in recent years with the aim of evaluating long-term outcomes of SBRT in oligometastatic NSCLC. A number phase I or II trials have been conducted as well in order to investigate the feasibility, safety, and efficacy of SBRT in patients with oligometastasis from NSCLC. All of them have shown that SBRT is feasible and safe in selected patient populations with favorable local control rates and treatment outcomes $(13,48-51)$. One notable trial is the randomized
Stereotactic Ablative Radiotherapy for the Comprehensive Treatment of Oligometastatic Disease (SABR-COMET) trial which enrolled 99 patients with controlled primary solid tumors and up to 5 metastatic lesions. Patients were randomized to standard of care (SOC) or to SOC + SBRT to all metastatic lesions. OS was 28 months in the SOC arm and 41 months in the intervention arm $(\mathrm{P}=0.09)$. PFS was 6 months in the SOC arm and 12 months in the intervention $\operatorname{arm}(\mathrm{P}=0.001)(52)$. The phase III SABR-COMET-10 is currently investigating the impact of SBRT in patients with 4-10 metastatic cancer lesions. Other notable ongoing trials are the NRG-LU002 and ETOP CHESS trials. The randomized phase II/III NRG-LU002 will assess the role of consolidative ablative therapies on OS. The multi-center single arm phase II ETOP CHESS-trial aims to assess the efficacy of immunotherapy, chemotherapy plus SBRT to oligometastases followed by definitive surgery or RT to the locoregional primary tumor in patients with histologicallyconfirmed synchronous oligometastatic NSCLC (53). Several other trials investigating the effects of SBRT and/or surgery on oligometastatic NSCLC are still ongoing. An overview of these trials is found in Table 2. However, despite an increasing number of promising studies, no RCTs comparing SBRT to surgery have been published as of yet. This means that, for 

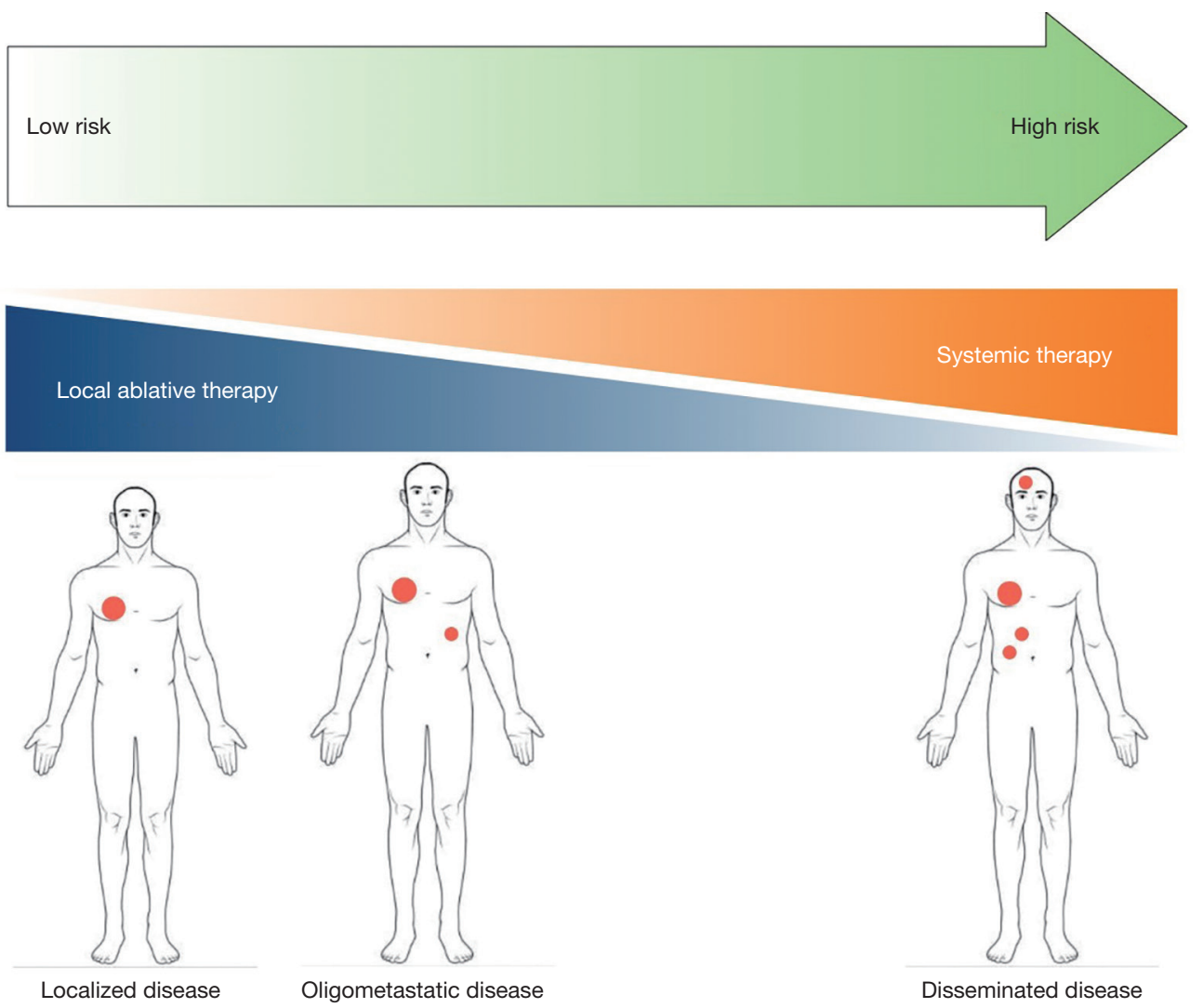

Figure 2 Overview of the spectrum theory of malignant diseases showing oligometastatic disease as an intermediate state between localized and disseminated disease. The benefit of local ablative therapy gradually decreases as the disease state progresses along the spectrum, while the reverse is seen for systemic treatments. In addition, progression along the spectrum is associated with higher risk diseases.

the foreseeable future, treatment strategies must be based on individual prognostic factors and require a rigorous work-up and a multidisciplinary approach. In the future, integration of clinical and molecular staging will hopefully allow a more personalized treatment approach across the wide spectrum of (oligo)metastatic diseases (Figure 2). More data from multi-center prospective trials will likely shed light on which therapeutic modalities are most suitable for the heterogeneous population of patients with oligometastatic NSCLC.

\section{Acknowledgments}

Funding: None.

\section{Footnote}

Provenance and Peer Review: This article was commissioned by the Guest Editors (Maurizio Infante \& Thierry Berghmans) for the series "Oligometastatic NSCLC: definition and treatment opportunities" published in Translational Lung Cancer Research. The article has undergone external peer review.

Reporting Checklist: The authors have completed the Narrative Review reporting checklist. Available at https:// dx.doi.org/10.21037/tlcr-21-58

Conflicts of Interest: All authors have completed the ICMJE uniform disclosure form (available at https://dx.doi. org/10.21037/tlcr-21-58). The series "Oligometastatic NSCLC: definition and treatment opportunities" was commissioned by the editorial office without any funding or sponsorship. PEVS serves as an unpaid editorial board member of Translational Lung Cancer Research from July 2019 to July 2021. The authors have no other conflicts of interest to declare.

Ethical Statement: The authors are accountable for all 
aspects of the work in ensuring that questions related to the accuracy or integrity of any part of the work are appropriately investigated and resolved.

Open Access Statement: This is an Open Access article distributed in accordance with the Creative Commons Attribution-NonCommercial-NoDerivs 4.0 International License (CC BY-NC-ND 4.0), which permits the noncommercial replication and distribution of the article with the strict proviso that no changes or edits are made and the original work is properly cited (including links to both the formal publication through the relevant DOI and the license). See: https://creativecommons.org/licenses/by-nc-nd/4.0/.

\section{References}

1. Ferlay J, Ervik M, Lam F, et al. Global Cancer Observatory: Cancer Today. Lyon, France: International Agency for Research on Cancer, 2020. Available online: https://gco.iarc.fr/today. Accessed 05/01/2021 2021.

2. Jemal A, Bray F, Center MM, et al. Global cancer statistics. CA Cancer J Clin 2011;61:69-90.

3. Ferlay J, Steliarova-Foucher E, Lortet-Tieulent J, et al. Cancer incidence and mortality patterns in Europe: estimates for 40 countries in 2012. Eur J Cancer 2013;49:1374-403.

4. Goldstraw P, Chansky K, Crowley J, et al. The IASLC Lung Cancer Staging Project: Proposals for Revision of the TNM Stage Groupings in the Forthcoming (Eighth) Edition of the TNM Classification for Lung Cancer. J Thorac Oncol 2016;11:39-51.

5. Juan O, Popat S. Ablative Therapy for Oligometastatic Non-Small Cell Lung Cancer. Clin Lung Cancer 2017;18:595-606.

6. Fois SS, Paliogiannis P, Zinellu A, et al. Molecular Epidemiology of the Main Druggable Genetic Alterations in Non-Small Cell Lung Cancer. Int J Mol Sci 2021;22:612.

7. Rolfo C, Castiglia M, Perez A, et al. Liquid Biopsy in Non-Small Cell Lung Cancer (NSCLC). In: Russo A, Giordano A, Rolfo C, et al. Liquid Biopsy in Cancer Patients: The Hand Lens for Tumor Evolution. Springer, 2017:103-15.

8. Zhuang $\mathrm{X}$, Zhao C, Li J, et al. Clinical features and therapeutic options in non-small cell lung cancer patients with concomitant mutations of EGFR, ALK, ROS1, KRAS or BRAF. Cancer Med 2019;8:2858-66.

9. Couñago F, Luna J, Guerrero LL, et al. Management of oligometastatic non-small cell lung cancer patients: Current controversies and future directions. World J Clin Oncol 2019;10:318-39.

10. Eberhardt WE, Mitchell A, Crowley J, et al. The IASLC Lung Cancer Staging Project: Proposals for the Revision of the M Descriptors in the Forthcoming Eighth Edition of the TNM Classification of Lung Cancer. J Thorac Oncol 2015;10:1515-22.

11. Ashworth AB, Senan S, Palma DA, et al. An individual patient data metaanalysis of outcomes and prognostic factors after treatment of oligometastatic non-small-cell lung cancer. Clin Lung Cancer 2014;15:346-55.

12. Gomez DR, Blumenschein GR Jr, Lee JJ, et al. Local consolidative therapy versus maintenance therapy or observation for patients with oligometastatic non-smallcell lung cancer without progression after first-line systemic therapy: a multicentre, randomised, controlled, phase 2 study. Lancet Oncol 2016;17:1672-82.

13. Iyengar P, Wardak Z, Gerber DE, et al. Consolidative Radiotherapy for Limited Metastatic Non-Small-Cell Lung Cancer: A Phase 2 Randomized Clinical Trial. JAMA Oncol 2018;4:e173501.

14. Hellman S, Weichselbaum RR. Oligometastases. J Clin Oncol 1995;13:8-10.

15. West H. Management of Oligometastatic Disease in Advanced Non-Small Cell Lung Cancer. Clin Chest Med 2020;41:249-58.

16. Foster CC, Pitroda SP, Weichselbaum RR. Definition, Biology, and History of Oligometastatic and Oligoprogressive Disease. Cancer J 2020;26:96-9.

17. Khodarev NN, Pitroda SP, Weichselbaum RR. microRNAs and oligometastasis. Aging (Albany NY) 2015;7:146-7.

18. Lussier YA, Xing HR, Salama JK, et al. MicroRNA expression characterizes oligometastasis(es). PLoS One 2011;6:e28650.

19. Korpal M, Kang Y. The emerging role of miR-200 family of microRNAs in epithelial-mesenchymal transition and cancer metastasis. RNA Biol 2008;5:115-9.

20. Lussier YA, Khodarev NN, Regan K, et al. Oligo- and polymetastatic progression in lung metastasis(es) patients is associated with specific microRNAs. PLoS One 2012;7:e50141.

21. Uppal A, Wightman SC, Mallon S, et al. 14q32-encoded microRNAs mediate an oligometastatic phenotype. Oncotarget 2015;6:3540-52.

22. Uppal A, Ferguson MK, Posner MC, et al. Towards a molecular basis of oligometastatic disease: potential role of micro-RNAs. Clin Exp Metastasis 2014;31:735-48. 
23. Pass HI, Carbone DP, Johnson DH, et al. Principles and Practice of Lung Cancer: the official reference text of the International Association for the Study of Lung Cancer (IASLC). Lippincott Williams \& Wilkins, 2012.

24. Lemjabbar-Alaoui H, Hassan OU, Yang YW, et al. Lung cancer: Biology and treatment options. Biochim Biophys Acta 2015;1856:189-210.

25. Bergsma DP, Salama JK, Singh DP, et al. Radiotherapy for Oligometastatic Lung Cancer. Front Oncol 2017;7:210.

26. Lanuti M. Surgical Management of Oligometastatic Non-Small Cell Lung Cancer. Thorac Surg Clin 2016;26:287-94.

27. Levy A, Hendriks LEL, Berghmans T, et al. EORTC Lung Cancer Group survey on the definition of NSCLC synchronous oligometastatic disease. Eur J Cancer 2019;122:109-14.

28. Palma DA, Salama JK, Lo SS, et al. The oligometastatic state - separating truth from wishful thinking. Nat Rev Clin Oncol 2014;11:549-57.

29. Giaj-Levra N, Giaj-Levra M, Durieux V, et al. Defining Synchronous Oligometastatic Non-Small Cell Lung Cancer: A Systematic Review. J Thorac Oncol 2019;14:2053-61.

30. Dingemans AC, Hendriks LEL, Berghmans T, et al. Definition of Synchronous Oligometastatic Non-Small Cell Lung Cancer-A Consensus Report. J Thorac Oncol 2019;14:2109-19.

31. Planchard D, Popat S, Kerr K, et al. Metastatic non-small cell lung cancer: ESMO Clinical Practice Guidelines for diagnosis, treatment and follow-up. Ann Oncol 2018;29:iv192-iv237. Erratum in: Ann Oncol. 2019 May;30(5):863-870. doi: 10.1093/annonc/mdy474. Epub 2019 Dec 4.

32. deSouza NM, Liu Y, Chiti A, et al. Strategies and technical challenges for imaging oligometastatic disease: Recommendations from the European Organisation for Research and Treatment of Cancer imaging group. Eur J Cancer 2018;91:153-63.

33. Schoenmaekers J, Hofman P, Bootsma G, et al. Screening for brain metastases in patients with stage III nonsmall-cell lung cancer, magnetic resonance imaging or computed tomography? A prospective study. Eur J Cancer 2019;115:88-96.

34. Volpi S, Ali JM, Tasker A, et al. The role of positron emission tomography in the diagnosis, staging and response assessment of non-small cell lung cancer. Ann Transl Med 2018;6:95.

35. Dinan MA, Curtis LH, Carpenter WR, et al. Stage migration, selection bias, and survival associated with the adoption of positron emission tomography among medicare beneficiaries with non-small-cell lung cancer, 1998-2003. J Clin Oncol 2012;30:2725-30.

36. Tönnies S, Tönnies M, Kollmeier J, et al. Impact of preoperative 18F-FDG PET/CT on survival of resected mono-metastatic non-small cell lung cancer. Lung Cancer 2016;93:28-34.

37. Li S, Zhu R, Li D, et al. Prognostic factors of oligometastatic non-small cell lung cancer: a meta-analysis. J Thorac Dis 2018;10:3701-13.

38. Ashworth A, Rodrigues G, Boldt G, et al. Is there an oligometastatic state in non-small cell lung cancer? A systematic review of the literature. Lung Cancer 2013;82:197-203.

39. Suzuki H, Yoshino I. Approach for oligometastasis in non-small cell lung cancer. Gen Thorac Cardiovasc Surg 2016;64:192-6.

40. Silvestri GA, Gonzalez AV, Jantz MA, et al. Methods for staging non-small cell lung cancer: Diagnosis and management of lung cancer, 3rd ed: American College of Chest Physicians evidence-based clinical practice guidelines. Chest 2013;143:e211S-e250S.

41. Bironzo P, Di Maio M. A review of guidelines for lung cancer. J Thorac Dis 2018;10:S1556-63.

42. Suzuki S, Goto T. Role of Surgical Intervention in Unresectable Non-Small Cell Lung Cancer. J Clin Med 2020;9:3881.

43. Wang Z, Gao SG, Xue Q, et al. Surgery of primary nonsmall cell lung cancer with oligometastasis: analysis of 172 cases. J Thorac Dis 2018;10:6540-6.

44. Yu HA, Sima CS, Huang J, et al. Local therapy with continued EGFR tyrosine kinase inhibitor therapy as a treatment strategy in EGFR-mutant advanced lung cancers that have developed acquired resistance to EGFR tyrosine kinase inhibitors. J Thorac Oncol 2013;8:346-51.

45. Weickhardt AJ, Scheier B, Burke JM, et al. Local ablative therapy of oligoprogressive disease prolongs disease control by tyrosine kinase inhibitors in oncogeneaddicted non-small-cell lung cancer. J Thorac Oncol 2012;7:1807-14.

46. Otake S, Goto T. Stereotactic Radiotherapy for Oligometastasis. Cancers (Basel) 2019;11:133.

47. Wujanto C, Vellayappan B, Siva S, et al. Stereotactic Body Radiotherapy for Oligometastatic Disease in Non-small Cell Lung Cancer. Front Oncol 2019;9:1219.

48. Collen C, Christian N, Schallier D, et al. Phase II study of stereotactic body radiotherapy to primary tumor and 
metastatic locations in oligometastatic nonsmall-cell lung cancer patients. Ann Oncol 2014;25:1954-9.

49. De Ruysscher D, Wanders R, Hendriks LE, et al. Progression-Free Survival and Overall Survival Beyond 5 Years of NSCLC Patients With Synchronous Oligometastases Treated in a Prospective Phase II Trial (NCT 01282450). J Thorac Oncol 2018;13:1958-61.

50. Iyengar P, Kavanagh BD, Wardak Z, et al. Phase II trial of stereotactic body radiation therapy combined with erlotinib for patients with limited but progressive metastatic non-small-cell lung cancer. J Clin Oncol 2014;32:3824-30.

51. Palma DA, Olson RA, Harrow S, et al. Stereotactic Ablative Radiation Therapy for the Comprehensive

Cite this article as: Berzenji L, Debaenst S, Hendriks JMH, Yogeswaran SK, Lauwers P, Van Schil PE. The role of the surgeon in the management of oligometastatic non-small cell lung cancer: a literature review. Transl Lung Cancer Res 2021;10(7):3409-3419. doi: 10.21037/tlcr-21-58
Treatment of Oligometastatic Tumors (SABR-COMET): Results of a Randomized Trial. Int J Radiat Oncol Biol Phys 2018;102:S3-4.

52. Palma DA, Olson R, Harrow S, et al. Stereotactic ablative radiotherapy versus standard of care palliative treatment in patients with oligometastatic cancers (SABRCOMET): a randomised, phase 2, open-label trial. Lancet 2019;393:2051-8.

53. Guckenberger M, Opitz I, Dafni U, et al. 1417TiP Immunotherapy, chemotherapy and stereotactic radiotherapy to metastases, followed by definitive surgery or radiotherapy to the primary tumour, in patients with synchronous oligo-metastatic NSCLC: The ETOP CHESS trial. Ann Oncol 2020;31:S895-6. 